\title{
Performance of clear vacuum-formed thermoplastic retainers depending on retention protocol: a systematic review
}

\author{
Eleftherios G. Kaklamanos ${ }^{1}\left(\right.$ C $^{-}$Maria Kourakou $^{2} \cdot$ Dimitrios Kloukos $^{2,3}$ • \\ Ioannis Doulis ${ }^{4} \cdot$ Smaragda Kavvadia $^{4}$
}

Received: 25 November 2015/Accepted: 27 April 2016/Published online: 6 June 2016

(C) The Society of The Nippon Dental University 2016

\begin{abstract}
We aimed at comparing the performance of vacuum-formed thermoplastic retainers (VFR) worn either full-time or part-time, in maintaining orthodontic treatment results in terms of tooth alignment, arch form and occlusion. We reviewed randomized and prospective controlled clinical trials comparing VFR wearing protocols and searched databases, without restrictions, for published and unpublished literature. The risk of bias was assessed using the Cochrane Risk of Bias tool and the overall level of certainty in the evidence following ADA methodology. 184 studies were initially identified and reduced to the 3 randomized controlled trials included in the systematic review by means of specific criteria. One study followed patients 1 year into retention, and the other two for 6 months. Little's Irregularity Index, intermolar and intercanine width, arch length, overjet and PAR score did not differ significantly between the patients wearing their retainers part time or full time. We observed a slight increase in the overbite in the part-time group in only one trial. With a moderate level of certainty, we found that during the
\end{abstract}

Eleftherios G. Kaklamanos

eleftherios.kaklamanos@mbru.ac.ae;

kaklamanos@yahoo.com

1 Hamdan Bin Mohammed College of Dental Medicine, Mohammed Bin Rashid University of Medicine and Health Sciences, Dubai Healthcare City, P.O. Box 505055, Dubai, United Arab Emirates

2 Department of Orthodontics and Dentofacial Orthopedics, 251 Hellenic Air Force V.A. General Hospital, Athens, Greece

3 Department of Orthodontics and Dentofacial Orthopedics, Faculty of Medicine, University of Bern, Bern, Switzerland

4 Department of Orthodontics, Aristotle University of Thessaloniki, Thessaloniki, Greece observation period, full-time VFR wear is not superior to part-time, bearing in mind the potential implications for health burden, retainer longevity and cost-effectiveness, as well as patient satisfaction and compliance.

Keywords Systematic review - Orthodontics - Retainers · Thermoplastic $\cdot$ Clear $\cdot$ Vacuum-formed

\section{Introduction}

After active orthodontic treatment, teeth show the tendency to return to their original position [1-3]. Thus, retention represents a fundamental phase among orthodontic procedures for almost every patient and, retainers are used to maintain teeth in their corrected position, opposing the influence from periodontal tissue stretching, pressures from orofacial muscle function, excessive or inadequate occlusal forces and continuing craniofacial growth [1-8]. These factors, together with parameters related to the nature of treatment and appliance selection, interact to force teeth back toward the pre-treatment position; a phenomenon observed in the vast majority of orthodontic patients $[2,3,5,6]$.

Fixed wire retainers and removable appliances, usually acrylic plates (Hawley or Begg-type) and thermoplastic vacuum-formed retainers (VFRs) are used to counteract any tendency to relapse [1-3, 6]. Removable appliances are better with regards to oral hygiene [9] but require patients' compliance [10, 11]. VFRs enjoy a variable but growing popularity among orthodontists, especially for use on the upper dental arch [12-18]. They are cost-effective compared to other types of removable appliances [19] more aesthetic, comfortable, less embarrassing and preferable from the patients' point of view [19-24]. For these reasons 
some patients are more likely to be compliant with the requirements of VFRs than with other types of removable appliances [22]. Although concerns on their durability exist, VFRs have proven similar to or even better than Hawley-type appliances with regards to fractures [19, 25].

The total period and the daily routine of VFR use that orthodontists advise their patients to comply with vary extensively [5]. Although animal observations determined that periodontal reorganization occurs after an average of 230 days [26, 27], protocols exist in the literature detailing varying extent and combinations of full-time (nearly $24 \mathrm{~h}$ per day) or part-time use [23, 28, 29]. So the variation in clinicians' suggestions is to be expected [14-16, 30] and this seems to be based more on preference, experience and other criteria than evidenced based research [30, 31].

Assuming that a retention protocol involving significantly less than wear for $24 \mathrm{~h}$ per day was effective in maintaining long-term stability in terms of tooth alignment, arch dimensions and occlusion after treatment, potential advantages could accrue regarding hard and soft oral tissues health, retainer longevity and cost-effectiveness, as well as patient satisfaction and their diligence in overall compliance to the proposed schedule [10, 14, 19, 21, 32]. The aim of the present systematic review was to investigate whether different VFR wearing protocols perform differently in maintaining the therapeutic result in subjects after orthodontic treatment.

\section{Materials and methods}

We carried out the present systematic review according to a pre-existing protocol following the guidelines outlined in the PRISMA statement [33] and the Cochrane Handbook for Systematic Reviews of Interventions (version 5.1.0) [34]. No funding was received for the present project.

\section{Selection criteria applied for the review}

Randomized (RCTs) and prospective controlled clinical trials (CCTs) were reviewed, comparing VFR wearing protocol effectiveness in maintaining orthodontic treatment result. The studies had to fulfil certain criteria regarding participants' and intervention characteristics, as well as principal outcome measures.

- Types of participants: patients referred for retention with thermoplastic vacuum-formed retainers after orthodontic treatment of any type. We placed no restrictions on the age of the patients.

- Types of interventions: we placed no restriction on the material of the thermoplastic retainer or the proposed wearing schedule.
- Outcome measures: we reviewed studies including measures that reflected changes in teeth alignment, arch form and occlusion, such as Irregularity Index [4], intercanine and intermolar width, arch length, overbite, overjet, PAR score [35, 36].

From this systematic review, we excluded studies reporting other types of orthodontic retainers, whether removable or not, animal studies, case reports, case series, systematic reviews and meta-analyses.

\section{Search strategy for identification of studies}

One of the authors (DK) developed detailed search strategies for each database searched. They were based on the strategy developed for MEDLINE but revised appropriately for each database to take account of the differences in controlled vocabulary and syntax rules. The following electronic databases were searched: MEDLINE (via Ovid and PubMed; Appendix 1) (1946 to 31 August 2014), EMBASE (via Ovid), the Cochrane Oral Health Group's Trials Register and CENTRAL. Unpublished literature was searched on ClinicalTrials.gov, the National Research Register, and ProQuest Dissertation and Theses Global database.

We placed no restriction on the language or date of publication, and searched the reference lists of all eligible studies for additional studies. Where additional information for some publication was needed, we contacted the respective authors.

\section{Selection of studies and data extraction}

Two reviewers (D.K. and M.K.) assessed the retrieved records for inclusion independently. They were not blinded to the identity of the authors, their institution, or the results of the research. They obtained and assessed, again independently, the full report of publications considered by either reviewer to meet the inclusion criteria. Disagreements were resolved by discussion or consultation with a third author (E.G.K.). We kept a record of all decisions on study identification.

The same two authors performed data extraction independently and any disagreements were again resolved by discussion or consultation with a third author (E.G.K.). We used data collection forms to record the desired information, such as bibliographic details, details on study design, verification of study eligibility, participant and intervention characteristics, patients' attrition and respective reasons, type of outcomes assessed and assessment procedures. We classified the reported wear regimens as part-time or fulltime according to authors' descriptions and clinical judgement. We used, if possible, mean differences and 
standard deviations to summarize continuous outcome data following a normal distribution, or the median and the interquartile range if they did not. We considered the patient as the unit of analysis for the outcomes related to occlusion changes (i.e., overbite, overjet, PAR score, etc.), and the jaw for the parameters related to tooth alignment and arch form (i.e., irregularity index, intercanine and intermolar width, arch length, etc.). Apart from outcomes related to treatment stability, we extracted any other possible relevant measures regarding patient reported outcomes, compliance, data on retainer condition (like thickness and integrity), retainer longevity, hard and soft oral tissue health and possible adverse effects. If we needed clarification on the published data or additional material, then we contacted the corresponding authors.

\section{Data synthesis and assessment of publication bias}

We planned to undertake a meta-analysis only if the retrieved studies presented adequate clinical and methodological homogeneity and reported similar measurements in appropriate statistical forms. In such cases, and if we identified an adequate number of studies, we planned to carry out analyses for "small-study effects" and publication bias.

\section{Assessment of risk of bias and determination of the level of certainty in the evidence}

Two review authors (M.K. and I.D.) assessed the risk of bias in the included studies, independently, using The Cochrane Collaboration's tool for RCTs [34], as no CCTs were finally found to be eligible for inclusion. Any disagreements were resolved by discussion or consultation with a third author (E.G.K.). The domains examined included random sequence generation, allocation concealment, blinding of participants and personnel, blinding of outcome assessors, incomplete outcome data, selective outcome reporting and other sources of bias. Each domain received a judgement of low, high or unclear risk of bias (indicating either lack of sufficient information to make a judgement or uncertainty over the risk of bias). Studies were finally grouped into the categories of low, unclear and high risk of bias.

The level of certainty in the evidence was assessed as high, moderate, or low according to the ADA Clinical Practice Guideline methodology that takes into account the parameters of the quantity of evidence, risk of bias, consistency, applicability, precision and publication bias [37].

\section{Results}

The flow of records through the reviewing process is shown in Fig. 1. We initially identified 184 references, and excluded 31 as duplicates and 113 more on the basis of their title and abstract. From the 40 records that remained, we excluded 8 because they involved surveys of retention protocols among orthodontists and 4 as literature reviews. Subsequently, we excluded another 25 records on the basis of the their full-text for various reasons (17 articles not referring to retention, 3 studies assessing patient compliance, 3 studies utilizing thermoplastic aligners and not retainers and 2 studies investigating Hawley retainers).

Finally, 3 full-text trial reports were included in the systematic review [38-40]. We made attempts to contact the corresponding authors of all three included studies for clarifications or additional unpublished data but none replied.

\section{Study characteristics and risk of bias assessment}

The characteristics of the studies included in the present systematic review are presented in Tables 1 and 2 . The papers were published between 2007 and 2012, had recruited 191 patients and analyzed 171. As no eligible CCTs could be retrieved, all included studies were RCTs. In the Thickett and Power [39] and Gill et al. [38] study the randomization procedure was based on patients, whereas in the Jäderberg et al. [40] publication randomization was based on jaws and consequently, a patient could be randomized to different wear regimen for the upper and lower retainer. Only the Thickett and Power study [39] followed patients 1 year into retention, with the others following patients for 6 months [38, 40]. All studies reported a priori calculating of sample size and dropouts, but no author carried out an intention-to-treat analysis.

The included studies involved diverse VFRs wearing protocols. Regarding the full-time wearing groups, only in the Gill et al. trial [38], did patients wear their VFRs $24 \mathrm{~h}$ per day for 6 months. In the other two studies, $24 \mathrm{~h} /$ day wearing was confined to the first trimester of observation and then retainer wear was reduced. In particular, in the Thickett and Power study [39], participants wore their retainers according to the following schedule: $0-3$ months: $24 \mathrm{~h}$ per day; 3-6 months: $10 \mathrm{~h}$ per day; 6-9 months: alternate nights; 9-12 months: 1-2 times per week. Finally, in Jäderberg et al. RCT patients wore the retainers $24 \mathrm{~h}$ per day for 3 months and only at night for the next 3 [40].

The part-time wearing protocols involved VFRs use for approximately $8-10 \mathrm{~h}$ per day in the first 6 months. In 
Fig. 1 Flow of records through the reviewing process
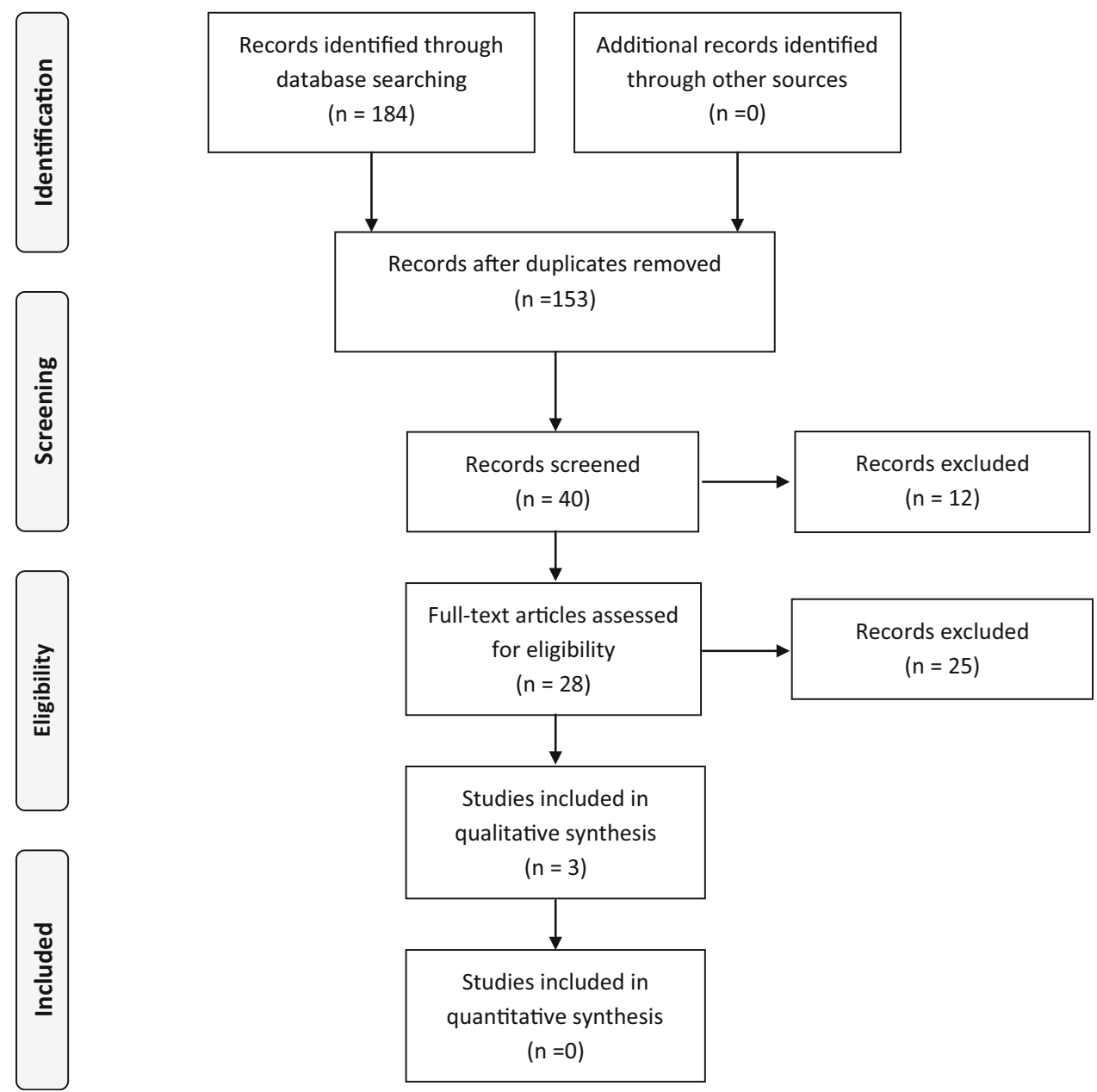

particular, in the Gill et al. investigation, patients were instructed to wear their retainers only during sleep (at least $8 \mathrm{~h}$ per day) for 6 months [38]. In the Thickett and Power study [39], patients followed the following scheme: 0-6 months: $10 \mathrm{~h}$ per day; 6-9 months: alternate nights; 9-12 months: 1-2 times per week. In the most recent study by Jäderberg et al., patients were told to wear their retainers $24 \mathrm{~h}$ per day only for the first week and then only at night until the end of the 6-month period [40].

All included studies evaluated changes in overjet (OJ), overbite (OB) and Little's Irregularity Index (LLI) [4]. Moreover, changes in intermolar and intercanine widths were evaluated in two studies [38, 39] and changes in arch length and PAR score $[35,36]$ were measured only in one [39]. Patient-reported outcomes in the form of a questionnaire were only assessed in one study [40].

Table 3 presents the summary findings of the risk of bias assessment for the included studies. All available studies were classified as presenting an overall unclear risk of bias, mainly because of uncertainties regarding the domains of random sequence generation and allocation concealment. However, it was felt much of the uncertainty might be due to poor reporting as other reports from the same investigators clearly describe the use of procedures with low risk of bias. Blinding of the participants could not be feasible because patients cannot be blinded upon the intervention. On the other hand, blinding of personnel could be possible but was not reported. Overall, in the context of the present research designs, there is no reason to believe that bias could be introduced because of absence of blinding in these cases. On the contrary, investigation of the procedures regarding blinding of the outcome assessment revealed unclear risk for one of the trials included [39]. Furthermore, in two studies, the dropouts and the respective reasons were described in details [38, 40], whereas the third did not present information on the cause of dropout but this deficiency was not deemed to introduce bias in the present context [39]. All three studies were considered of low risk regarding reporting bias.

\section{Comparison between wear schedules}

The results of the studies included in this systematic review are summarized in Table 4 . We were not able to perform 


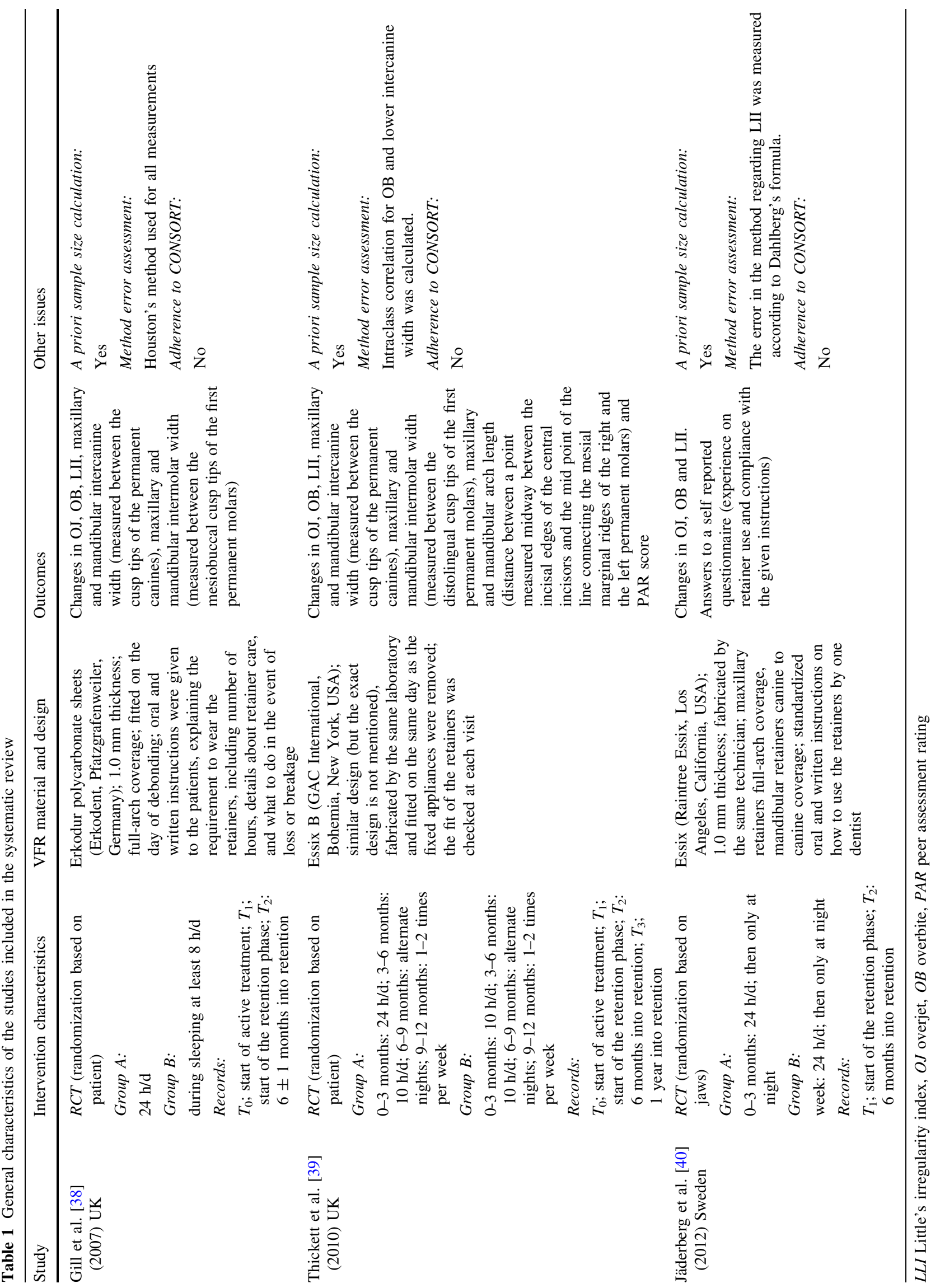




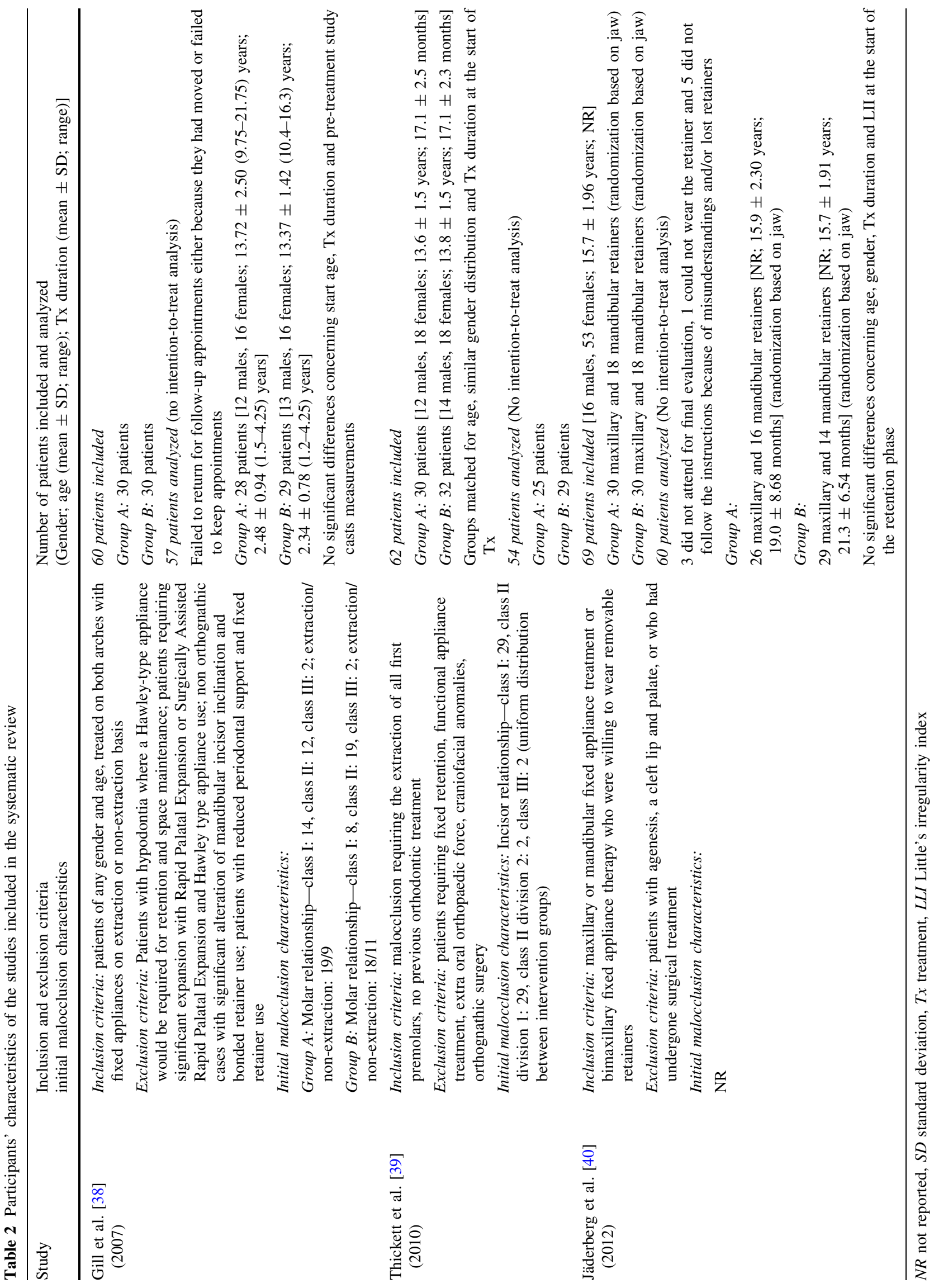


Table 3 Summary of risk of bias assessment

\begin{tabular}{llll}
\hline Domain & \multicolumn{2}{l}{ Study } & \\
\cline { 2 - 4 } & Gill et al. 2007 [38] & Thickett et al. 2010 [39] & Jäderberg et al. 2012 [40] \\
\hline Random sequence generation & Unclear risk & Unclear risk & Unclear risk \\
Allocation concealment & Unclear risk & Unclear risk & Unclear risk \\
Blinding of participants and personnel & Low risk & Low risk & Low risk \\
Blinding of outcome assessment & Low risk & Unclear risk & Low risk \\
Incomplete outcome data & Low risk & Low risk & Low risk \\
Selective outcome reporting & Low risk & Low risk & Low risk \\
Other potential threats to validity & Low risk & Unclear risk & Low risk \\
Summary assessment of within study risk of bias & Unclear risk for key domains & Unclear risk for key domains & Unclear risk for key domains
\end{tabular}

Table 4 Summary of comparisons ( $p$ values) between the various VFRs' wear regimens

\begin{tabular}{|c|c|c|c|c|}
\hline \multirow[t]{2}{*}{ Study } & \multicolumn{3}{|c|}{ Observation at 6 months } & \multirow{2}{*}{$\begin{array}{l}\text { Observation at } 12 \text { months } \\
\text { Thickett and Power } 2010 \text { [39] }\end{array}$} \\
\hline & Gill et al. $2007[38]^{\mathrm{a}}$ & Thickett and Power $2010[39]^{\mathrm{b}}$ & Jäderberg et al. $2012[40]^{\mathrm{c}}$ & \\
\hline \multicolumn{5}{|l|}{ Maxillary measurements } \\
\hline Little's irregularity index & 0.60 & 0.67 & $>0.05$ & 0.80 \\
\hline Intercanine width & 0.89 & 0.34 & - & 0.52 \\
\hline Intermolar width & 0.81 & 0.62 & - & 0.68 \\
\hline Arch length & - & 0.40 & - & 0.97 \\
\hline \multicolumn{5}{|l|}{ Mandibular measurements } \\
\hline Little's irregularity index & 0.93 & 0.08 & $>0.05$ & 0.50 \\
\hline Intercanine width & 0.56 & 0.31 & - & 0.65 \\
\hline Intermolar width & 0.74 & 0.69 & - & 0.61 \\
\hline Arch length & - & 0.14 & - & 0.06 \\
\hline \multicolumn{5}{|l|}{ Interarch measurements } \\
\hline Overjet & 0.80 & 0.55 & $>0.05$ & 0.37 \\
\hline Overbite & 0.11 & $\mathbf{0 . 0 2}$ (part time $>$ full time) & $>0.05$ & 0.05 (part time $>$ full time $)$ \\
\hline PAR score & - & $>0.05$ & - & $>0.05$ \\
\hline
\end{tabular}

Bold value indicate statistical significance

$P A R$ peer assessment rating, - not measured

${ }^{a}$ Differences in changes during the observation period (independent samples Student's $t$ test)

${ }^{\mathrm{b}}$ Differences in measurements at 6 months (Mann-Whitney $U$ test)

${ }^{c}$ Differences in changes during the observation period (Mann-Whitney $U$ test)

${ }^{d}$ Differences in measurements at 12 months (Mann-Whitney $U$ test)

meta-analysis and examine publication and reporting biases because of the lack of extensive relevant data, the differences in the interventions used, as well as, the variability in statistical reporting.

Overall, we observed no statistically significant difference between the compared VFR wearing protocols, with regards to Little's Irregularity Index [38-40], intermolar and intercanine width [38, 39], arch length [39], overjet [38-40] and PAR score [39]. In the Jäderberg and coworkers study [40], which also involved cases with only either maxillary or mandibular VFRs, no significant differences in the above measurements were observed, when these patients were compared to those having retainers in both arches. We judged the overall level of certainty in the evidence to be moderate for the abovementioned outcomes based on the evidence profile described by the ADA Clinical Practice Guideline [37].

Only regarding the parameter of overbite, did we note in one study [39] a statistically significant greater measurement in the "part-time" wearing group (0-6 months: $10 \mathrm{~h}$ per day; 6-9 months: alternate nights; 9-12 months: 1-2 times per week) compared to the "full-time" wearing 
group which differed in the fact that the appliance was worn for $24 \mathrm{~h}$ per day from 0 to 3 months. However, we did not observe similar differences in the other two studies included in this systematic review [38, 40]. Again, in the Jäderberg and co-workers study [40], that also involved cases with either maxillary or mandibular VFRs alone, no significant difference in overbite was observed, when these patients were compared to those having retainers in both arches. We judged the overall level of certainty in the evidence to be low for this outcome based on the evidence profile described by the ADA Clinical Practice Guideline [37].

Regarding patient-reported outcomes, although the Jäderberg et al. study [40] employed a questionnaire, comparisons between the two wear regimens were not presented in the report. Moreover, although it was measured in this trial, the thickness of the retainers was not compared between the two groups. Other recordings related to compliance, retainer integrity and longevity, measures on hard and soft oral tissue health and possible adverse effects were not found or compared between groups in the studies included in the present systematic review.

\section{Discussion}

In this systematic review that followed well-established guidelines, we concluded with a moderate level of evidence that we did not observe superiority of full-time VFR wearing protocols with regards to teeth alignment, arch dimensions and occlusion stability for the duration of the studies [38-40]. Only one trial [39] reported a slight statistically, but probably not clinically, significant increase in overbite in the part-time group.

The fact that only three trials satisfied the pre-specified inclusion criteria to be included in this systematic review reflects the scarcity of relevant research and the consequent lack of extensive data. Thankfully, those trials were RCTs, as it is widely accepted that well-designed and properly executed RCTs provide the best evidence on the effectiveness of health care interventions [41]. The shortage of VFR related research can be considered rather surprising as retention constitutes a fundamental phase of orthodontic procedures for almost every patient to counteract the environment created by the influence of muscle function, forces of occlusion and continuing craniofacial growth, as well as, various treatment-related parameters [1-8]. In addition, the paucity of scientific interest becomes more important as the popularity of VFRs increases [12-18]. Up to now, the protocols used in clinical practice seem to be guided primarily by operator preference and personal experience, as well as other criteria that are not compatible with evidence based practice [30, 31], and have resulted in a variety of empirical retention protocols employed by orthodontists [14-16, 30].

Based on the studies included in this systematic review, we did not observe significant differences in effectiveness between full and part-time VFRs wearing schedules concerning various measurements related to treatment result stability, such as Little's Irregularity Index, intermolar and intercanine width, arch length, overjet and PAR score [38-40]. The only notable exception to this trend involved overbite, which was found to have increased only in one trial in the 6 month measurements of the part-time wear group, and only by $0.77 \mathrm{~mm}(p=0.02)$ [39]. The authors of the report attributed this finding to more rapid settling. The same difference continued to exist in the 12-month recordings, but had diminished to $0.60 \mathrm{~mm}$ and barely reached statistical significance $(p=0.05)$. Nevertheless, overbite differences in both data measurements points do not seem to be a problem of clinical significance in most treatment cases. On the contrary, one advantage of parttime wear protocols may be that settling of teeth can occur unobstructed when the retainers are not worn [42]. Maximal centric stops in intercuspation are believed to be important in maintaining occlusal stability, distributing stresses among teeth evenly and ensuring tooth loading along the long axes [43].

Consequently, one could suggest with a moderate level of certainty that part-time VFR wear protocols (as the ones described in the included trials) could be sufficient in maintaining the result obtained after orthodontic treatment, with regards to teeth alignment, arch dimensions and occlusion stability. This finding could have immediate clinical implications, as the decreased appliance burden could demonstrate potential advantages, such as reduced risk of enamel demineralization and healthier periodontal tissues [32]. Part-time wear of a "less visible" retainer like VFR could also mean less impact on everyday activities and an increase in patient satisfaction, aesthetic perception and comfort. These parameters have been suggested to enhance overall compliance to the retention protocol being followed [10, 14, 19, 21]. Moreover, reduced wear could increase retainer longevity, in terms of fractures and loss of fit, and add another dimension to the demonstrated costeffectiveness of the intervention [19]. However, although extremely interesting, none of these parameters were studied or related recordings compared in the patient groups described in this systematic review along with the data on stability and further research is warranted in this respect.

In general, reducing clinical diversity and methodological variability, as well as, consistent and adequate reporting, could benefit future trials. For example, Jäderberg and co-workers [40] based randomization on jaws 
rather than on patients. Consequently, a patient could be randomized to different wear regimens group for the maxillary and mandibular retainer. This procedure could be really confusing for the patient and lead to an increase in the number of dropouts due to misunderstandings. Appropriate reporting could also resolve uncertainties during risk of bias assessment; in general, it was felt that much of this uncertainty could be attributed to poor reporting as other later studies from the same investigators clearly described the use of procedures with low risk of bias.

Longer follow-ups could also add to the robustness of relevant trials as the available data on the continued craniofacial growth [8] and post-treatment changes [44] underline the importance of prolonged (greater than 12 months) or even indefinite retention [1]. In addition, there is a need to take into account and assess objectively patients' compliance to the specified retention protocols $[10,11]$. Many parameters have been found to influence compliance, including intelligence, patient and doctor selfesteem, patient-doctor relationship, clear explanation to patients and parents of the rationale of retention and the risks associated with non compliance, as well as, frequent control visits [45-47]. Assessment of compliance can be very challenging, particularly for research purposes [48]. Subjective measurements, like patient completed forms, patients' and parents' reports, as well as clinicians' estimates are not considered reliable [48-51]. Devices used in the past for the objective measurement of compliance have proved to be expensive, complex, unreliable and inaccurate [47-49, 52, 53]. Lately, electronic micro-sensors have shown to be promising in objectively assess compliance, since they can be easily fitted onto removable appliances and measure accurately and reliably the exact period of appliance wearing [10, 54-58].

The aforementioned observed lack of more extended observation periods and objective assessment of patients' compliance constituted the major limitations of the material included in the present review. Second, clinical diversity, inconsistent reporting, non-response by the authors when asked for clarifications, as well as, the incapacity to examine publication and reporting biases precluded the formation of conclusions with a high level of certainty.

In summary, there exists a moderate level of certainty that part-time VFR wearing protocols could be sufficient in maintaining the result obtained after orthodontic treatment in the short-term. For clinical practice this finding could possibly result in decreased burden on hard and soft tissues health, increased retainer longevity and cost-effectiveness, as well as, patient satisfaction and overall compliance. However, standardization, better reporting in longer follow-ups and data on additional parameters, such as, patient-reported outcomes and integrity of retainers and longevity could be useful.

\section{Compliances with ethical standards}

Conflict of interest The authors declare that they have no conflict of interest.

\section{Appendix 1}

Search in Medline through PubMed, 31.08.2014.

\begin{tabular}{|c|c|c|}
\hline$\# 1$ & Orthodontic retention & 1285 \\
\hline \#2 & (Orthodontic retention) AND removable[Title/Abstract] & 112 \\
\hline \#3 & (Orthodontic retention) AND essix[Title/Abstract] & 11 \\
\hline \#4 & (Orthodontic retention) AND Vacuum[Title/Abstract] & 13 \\
\hline \#5 & $\begin{array}{l}\text { (Orthodontic retention) AND transparent[Title/ } \\
\text { Abstract] }\end{array}$ & 3 \\
\hline \#6 & (Orthodontic retention) AND splint[Title/Abstract] & 35 \\
\hline \#7 & $\begin{array}{l}\text { (Orthodontic retention) AND thermoplastic[Title/ } \\
\text { Abstract] }\end{array}$ & 10 \\
\hline \#8 & Orthodontic retainer & 1036 \\
\hline \#9 & (Orthodontic retainer) AND essix[Title/Abstract] & 25 \\
\hline \#10 & (Orthodontic retainer) AND removable[Title/Abstract] & 113 \\
\hline \#11 & (Orthodontic retainer) AND transparent[Title/Abstract] & 3 \\
\hline \#12 & (Orthodontic retainer) AND vacuum[Title/Abstract] & 22 \\
\hline \#13 & (Orthodontic retainer) AND splint[Title/Abstract] & 45 \\
\hline \#14 & $\begin{array}{l}\text { (Orthodontic retainer) AND thermoplastic[Title/ } \\
\text { Abstract] }\end{array}$ & 20 \\
\hline \#15 & Orthodontic retention[MeSH Major Topic] Schema: all & 0 \\
\hline \#16 & Orthodontic retainer[MeSH Terms] & 766 \\
\hline \#17 & $\begin{array}{l}\text { Orthodontic retainer[MeSH Terms]) AND Essix[Title/ } \\
\text { Abstract] }\end{array}$ & 21 \\
\hline \#18 & $\begin{array}{l}\text { (Orthodontic retainer[MeSH Terms]) AND splint[Title/ } \\
\text { Abstract] }\end{array}$ & 30 \\
\hline \#19 & $\begin{array}{l}\text { (Orthodontic retainer[MeSH Terms]) AND } \\
\text { removable[Title/Abstract] }\end{array}$ & 67 \\
\hline \#20 & $\begin{array}{l}\text { (Orthodontic retainer[MeSH Terms]) AND } \\
\text { transparent[Title/Abstract] }\end{array}$ & 2 \\
\hline \#21 & $\begin{array}{l}\text { (Orthodontic retainer[MeSH Terms]) AND } \\
\text { Vacuum[Title/Abstract] }\end{array}$ & 15 \\
\hline \#22 & $\begin{array}{l}\text { Orthodontic retainer[MeSH Terms]) AND } \\
\text { thermoplastic[Title/Abstract] }\end{array}$ & 17 \\
\hline \multirow[t]{2}{*}{ \#23 } & $\begin{array}{l}((\text { Orthodont*[Title/Abstract] }) \text { AND (Retention OR } \\
\text { retainer[Title/Abstract] }))\end{array}$ & 749 \\
\hline & NOT (Bonded OR fixed[Title/Abstract]) & \\
\hline \multirow[t]{2}{*}{ \#24 } & $\begin{array}{l}((((\text { Orthodont*[Title/Abstract] }) \text { AND (Retention OR } \\
\text { retainer[Title/Abstract] }))\end{array}$ & 8 \\
\hline & $\begin{array}{l}\text { NOT (Bonded OR fixed[Title/Abstract]))) AND } \\
\text { Essix[Title/Abstract] }\end{array}$ & \\
\hline \multirow[t]{2}{*}{ \#25 } & $\begin{array}{l}((((\text { Orthodont* } * \text { Title/Abstract] }) \text { AND (Retention OR } \\
\text { retainer[Title/Abstract }]))\end{array}$ & 7 \\
\hline & $\begin{array}{l}\text { NOT (Bonded OR fixed[Title/Abstract]))) AND } \\
\text { Vacuum[Title/Abstract] }\end{array}$ & \\
\hline \multirow[t]{2}{*}{ \#26 } & $\begin{array}{l}((((\text { Orthodont*[Title/Abstract] }) \text { AND (Retention OR } \\
\text { retainer[Title/Abstract] }))\end{array}$ & 1 \\
\hline & $\begin{array}{l}\text { NOT (Bonded OR fixed[Title/Abstract]))) AND } \\
\text { Transparent[Title/Abstract] }\end{array}$ & \\
\hline
\end{tabular}


\#27 ((((Orthodont*[Title/Abstract]) AND (Retention OR

retainer[Title/Abstract]))

NOT (Bonded OR fixed[Title/Abstract]))) AND removable[Title/Abstract]

\#28 (((Orthodont*[Title/Abstract]) AND (Retention OR retainer[Title/Abstract]))

NOT (Bonded OR fixed[Title/Abstract]))) AND splint[Title/Abstract]

\section{References}

1. Johnston C, Burden D, Morris D. Clinical guidelines: orthodontic retention (Revised by Parvizi F, Morris D, Atack N). London: British Orthodontic Society; 2013.

2. Joondeph DR. Stability, retention and relapse. In: Graber TM, Vanarsdall RL, Vig KWL (eds) Orthodontics. Current principles and techniques, 5th edn. Philadelphia: Elsevier; 2011.

3. Blake M, Garvey MT. Rationale for retention following orthodontic treatment. J Can Dent Assoc. 1998;64:640-1.

4. Little RM. The irregularity index: a quantitative score of mandibular anterior alignment. Am J Orthod. 1975;68:554-63.

5. Blake M, Bibby K. Retention and stability: a review of the literature. Am J Orthod Dentofac Orthop. 1998;114:299-306.

6. Melrose C, Millett DT. Toward a perspective on orthodontic retention? Am J Orthod Dentofac Orthop. 1998;113:507-14.

7. Ingervall B. Cheek pressure and head posture. Angle Orthod. 1988;58:47-57.

8. Sinclair PM, Little RM. Maturation of untreated normal occlusions. Am J Orthod. 1983;83:114-23.

9. Heier EE, De Smit AA, Wijgaerts IA, Adriaens PA. Periodontal implications of bonded versus removable retainers. Am J Orthod Dentofac Orthop. 1997;112:607-16.

10. Ackerman MB, Thornton B. Posttreatment compliance with removable maxillary retention in a teenage population: a shortterm randomized clinical trial. Orthod (Chic.). 2011;12:22-7.

11. Sheridan JJ. The three keys of retention. J Clin Orthod. 1991;25:717-8.

12. Meade MJ, Millett D. Retention protocols and use of vacuumformed retainers among specialist orthodontists. J Orthod. 2013;40:318-25

13. Vandevska-Radunovic V, Espeland L, Stenvik A. Retention: type, duration and need for common guidelines. A survey of Norwegian orthodontists. Orthodontics (Chic.). 2013;14:e110-7.

14. Pratt MC, Kluemper GT, Hartsfield JK Jr, Fardo D, Nash DA. Evaluation of retention protocols among members of the American Association of Orthodontists in the United States. Am J Orthod Dentofac Orthop. 2011;140:520-6.

15. Valiathan M, Hughes E. Results of a survey-based study to identify common retention practices in the United States. Am J Orthod Dentofac Orthop. 2010;137:170-7.

16. Renkema AM, Sips ET, Bronkhorst E, Kuijpers- Jagtman AM. A survey on orthodontic retention procedures in the Netherlands. Eur J Orthod. 2009;31:423-37.

17. Singh P, Grammati S, Kirschen R. Orthodontic retention patterns in the United Kingdom. J Orthod. 2009;36:115-21.

18. Wong PM, Freer TJ. A comprehensive survey of retention procedures in Australia and New Zealand. Aust Orthod J. 2004;20:99-106.

19. Hichens L, Rowland H, Williams A, Hollinghurst S, Ewings P, Clark S, Ireland A, Sandy J. Cost-effectiveness and patient

satisfaction: Hawley and vacuum-formed retainers. Eur J Orthod. 2007;29:372-8.

20. Wong P, Freer TJ. Patients' attitudes towards compliance with retainer wear. Aust Orthod J. 2005;21:45-53.

21. Sawhney B. Orthodontic retainers: a survey of patient compliance and satisfaction. Dissertation. London: University of Western Ontario; 2013.

22. Mollov ND, Lindauer SJ, Best AM, Shroff B, Tufekci E. Patient attitudes toward retention and perceptions of treatment success. Angle Orthod. 2010;80:468-73.

23. Lindauer SJ, Shoff RC. Comparison of Essix and Hawley retainers. J Clin Orthod. 1998;32:95-7.

24. Haydar B, Karabulut G, Ozkan S, Aksoy AU, Ciger S. Effects of retainers on the articulation of speech. Am J Orthod Dentofac Orthop. 1996;110:535-40.

25. Sun J, Yu YC, Liu MY, Chen L, Li HW, Zhang L, Zhou Y, Ao D, Tao R, Lai WL. Survival time comparison between Hawley and clear overlay retainers: a randomized trial. J Dent Res. 2011;90:1197-201.

26. Reitan K. Clinical and histological observations on tooth movement during and after orthodontic treatment. Am J Orthod. 1967;53:721-45.

27. Reitan K. Principles of retention and avoidance of posttreatment relapse. Am J Orthod. 1969;55:776-90.

28. Rowland H, Hichens L, Williams A, Hills D, Killingback N, Ewings P, Clark S, Ireland AJ, Sandy JR. The effectiveness of Hawley and vacuum-formed retainers: a single-center randomized controlled trial. Am J Orthod Dentofac Orthop. 2007;132:730-7.

29. Wang F. A new thermoplastic retainer. J Clin Orthod. 1997;31:754-7.

30. Lai CS, Grossen JM, Renkema AM, Bronkhorst E, Fudalej PS, Katsaros C. Orthodontic retention procedures in Switzerland. Swiss Dent J. 2014;124:655-61.

31. Clark JD, Kerr WJ, Davis MH. CASES—clinical audit; scenarios for evaluation and study. Br Dent J. 1997;183:108-11.

32. Sheridan JJ, Armbruster P, Moskowitz E, Nguyen P. Avoiding demineralization and bite alteration from full-coverage plastic appliances. J Clin Orthod. 2001;35:444-8.

33. Moher D, Liberati A, Tetzlaff J, Altman DG, The PRISMA Group. Preferred reporting items for systematic reviews and meta-analyses: The PRISMA statement. Open Med. 2001;3:123-130.

34. Higgins JPT, Green S. Cochrane handbook for systematic reviews of interventions version 5.1.0. The Cochrane Collaboration; 2011.

35. Richmond S, Shaw WC, O'Brien KD, Buchanan IB, Jones R, Stephens CD, Roberts CT, Andrews M. The development of the PAR Index (Peer Assessment Rating): reliability and validity. Eur J Orthod. 1992;14:125-39.

36. Richmond S, Shaw WC, Roberts CT, Andrews M. The PAR Index (Peer Assessment Rating): methods to determine outcome of orthodontic treatment in terms of improvement and standards. Eur J Orthod. 1992;14:180-7.

37. American Dental Association. ADA clinical practice guideline handbook: 2013 Update. Chicago: American Dental Association; 2013.

38. Gill DS, Naini FB, Jones A, Tredwin CJ. Part-time versus fulltime retainer wear following fixed appliance therapy: a randomized prospective controlled trial. World J Orthod. 2007;8:300-6.

39. Thickett E, Power S. A randomized clinical trial of thermoplastic retainer wear. Eur J Orthod. 2010;32:1-5.

40. Jäderberg S, Feldmann I, Engström C. Removable thermoplastic appliances as orthodontic retainers-a prospective study of different wear regimens. Eur J Orthod. 2012;34:475-9.

41. Altman DG, Schulz KF, Moher D, Egger M, Davidoff F, Elbourne D, Gøtzsche PC, Lang T, CONSORT GROUP 
(Consolidated Standards of Reporting Trials). The revised CONSORT statement for reporting randomized trials: explanation and elaboration. Ann Intern Med. 2001;134:663-94.

42. Aslan BI, Dinçer M, Salmanli O, Qasem MA. Comparison of the effects of modified and full-coverage thermoplastic retainers on occlusal contacts. Orthodontics (Chic.). 2013;14:e198-208.

43. Dinçer M, Meral O, Tümer N. The investigation of occlusal contacts during the retention period. Angle Orthod. 2003;73:640-6.

44. Little RM, Robert M. Little on the University of Washington post-retention studies. J Clin Orthod. 2009;43:723-7.

45. Mortensen MG, Kiyak HA, Omnell L. Patient and parent understanding of informed consent in orthodontics. Am J Orthod Dentofac Orthop. 2003;124:541-50.

46. Sergl HG, Klages U, Zentner A. Functional and social discomfort during orthodontic treatment-effects on compliance and prediction of patients' adaptation by personality variables. Eur J Orthod. 2000;22:307-15.

47. Bartsch A, Witt E, Sahm G, Schneider S. Correlates of objective patient compliance with removable appliance wear. Am J Orthod Dentofac Orthop. 1993;104:378-86.

48. Lee SJ, Ahn SJ, Kim TW. Patient compliance and locus of control in orthodontic treatment: a prospective study. Am J Orthod Dentofac Orthop. 2008;133:354-8.

49. Bos A, Kleverlaan CJ, Hoogstraten J, Prahl-Andersen B, Kuitert R. Comparing subjective and objective measures of headgear compliance. Am J Orthod Dentofac Orthop. 2007;132:801-5.
50. Brandao M, Pinho HS, Urias D. Clinical and quantitative assessment of headgear compliance: a pilot study. Am J Orthod Dentofac Orthop. 2006;129:239-44.

51. Kyriacou PA, Jones DP. Compliance monitor for use with removable orthodontic headgear appliances. Med Biol Eng Comput. 1997;35:57-60.

52. Cureton SL, Regennitter F, Orbell MG. An accurate, inexpensive headgear timer. J Clin Orthod. 1991;25:749-54.

53. Sahm G, Bartsch A, Witt E. Micro-electronic monitoring of functional appliance wear. Eur J Orthod. 1990;12:297-301.

54. Ackerman MB, McRae MS, Longley WH. Microsensor technology to help monitor removable appliance wear. Am J Orthod Dentofac Orthop. 2009;135:549-51.

55. Schäfer K, Ludwig B, Meyer-Gutknecht H, Schott TC. Quantifying patient adherence during active orthodontic treatment with removable appliances using microelectronic wear-time documentation. Eur J Orthod. 2015;37:73-80.

56. Schott TC, Göz G. Applicative characteristics of new microelectronic sensors Smart Retainer ${ }^{\circledR}$ and TheraMon ${ }^{\circledR}$ for measuring wear time. J Orofac Orthop. 2010;71:339-47.

57. Schott TC, Ludwig B, Glasl BA, Lisson JA. A microsensor for monitoring removable appliance wear. J Clin Orthod. 2011;45:518-20.

58. Schott TC, Ludwig B. Microelectronic wear-time documentation of removable orthodontic devices detects heterogeneous wear behavior and individualizes treatment planning. Am J Orthod Dentofac Orthop. 2014;146:155-60. 